\title{
Implicações da COVID-19 para pacientes gestantes
}

\author{
Implications of COVID-19 for pregnant patients
}

Implicaciones de COVID-19 para pacientes embarazadas

Lidiane Pereira de Albuquerque ${ }^{1 *}$, Ana Vitória Leite Monte ${ }^{2}$, Regina Maria Sousa de Araújo .

\section{RESUMO}

Objetivo: Destacar, através de uma revisão narrativa, o manejo e medidas de controle da COVID-19 para as gestantes e os possíveis riscos durante a transmissão vertical e a amamentação. Utilizaram-se bases de dados PubMed e Science Direct que retrataram artigos sobre infecções virais, COVID-19, gestação e amamentação. Revisão bibliográfica: Alterações fisiológicas, metabólicas e vasculares podem agravar a apresentação clínica da COVID-19, promovendo disfunção endotelial, ativação do sistema complemento e um efeito pró-trombótico nas gestantes. Não há relatos da detecção do novo coronavírus nos líquidos amniótico e placentário, sangue do cordão umbilical ou no leite materno. No entanto, alguns estudos sugerem que é possível a transmissão vertical do vírus e uma maior prevalência de partos prematuros tem sido notificada. $O$ tratamento inicia-se quando possíveis benefícios superam os potenciais riscos da terapia ao feto. Considerações finais: Procedimentos devem ser implementados para contornar as lacunas de conhecimento sobre o efeito da COVID-19 na gravidez, incluindo estratégias das vigilâncias sanitária, epidemiológica, pesquisas socioeconômicas e de soroprevalência.

Palavras-chave: COVID-19, Gravidez, Amamentação, Transmissão vertical.

\section{ABSTRACT}

Objective: Highlight, through a narrative review, the management and control measures of COVID-19 for pregnant women and the possible risks during vertical transmission and breastfeeding. PubMed and Science Direct databases were used, which portrayed articles on viral infections, COVID-19, pregnancy and breastfeeding. Bibliographic review: Physiological, metabolic and vascular disturbances can worsen the clinical presentation of COVID-19, promoting endothelial dysfunction, activation of the complement system, and a prothrombotic effect in pregnant women. There are no reports of the new coronavirus detection in amniotic and placental fluids, cord blood or breast milk. However, some studies suggest that vertical transmission of the virus is possible and a higher prevalence of premature births has been reported. Treatment begins when possible benefits outweigh the potential risks of therapy to the fetus. Final considerations: Procedures should be implemented to bridge knowledge gaps about the effect of COVID19 on pregnancy, including health surveillance, epidemiological, socioeconomic, and seroprevalence surveys.

Key words: COVID-19, Pregnancy, Breastfeeding, Vertical transmission.

\section{RESUMEN}

Objetivo: Destacar, a través de una revisión narrativa, las medidas de gestión y control de COVID-19 para mujeres embarazadas y los posibles riesgos durante la transmisión vertical y la lactancia. Se utilizaron las bases de datos PubMed y Science Direct, que retrataron artículos sobre infecciones virales, COVID-19, embarazo y lactancia. Revisión bibliográfica: Los cambios fisiológicos, metabólicos y vasculares pueden empeorar la presentación clínica de COVID-19, promoviendo la disfunción endotelial, la activación del

1Universidade Federal do Piauí (UFPI), Teresina - PI. *E-mail: lidianealbuquerque@ufpi.edu.br ${ }^{2}$ Centro Universitário UniFacid Wyden, Teresina - PI. 
sistema del complemento y un efecto protrombótico en mujeres embarazadas. No hay informes de la detección del nuevo coronavirus en fluidos amnióticos y placentarios, sangre del cordón umbilical o leche materna. Sin embargo, algunos estudios sugieren que la transmisión vertical del virus es posible y se ha informado una mayor prevalencia de nacimientos prematuros. El tratamiento comienza cuando los posibles beneficios superan los riesgos potenciales de la terapia para el feto. Consideraciones finales: Deben implementarse procedimientos para cerrar las brechas de conocimiento sobre el efecto de COVID-19 en el embarazo, incluidas las encuestas de vigilancia de la salud, epidemiológicas, socioeconómicas y de seroprevalencia.

Palabras clave: COVID-19, Embarazo, Amamantamiento, Transmisión vertical.

\section{INTRODUÇÃO}

A doença pelo novo coronavírus 2019 (COVID-19), causada pelo coronavírus (CoV) da síndrome respiratória aguda grave 2 (SARS-CoV-2), foi declarada como pandemia pela Organização Mundial da Saúde (OMS) em março de 2020 (WHO, 2020). SARS-CoV-2 faz parte de uma família de CoVs composta por outros quatro vírus que causam resfriado comum, além dos CoVs da Síndrome Respiratória Aguda Grave (SARS) e da Síndrome Respiratória do Oriente Médio (MERS).

Dados sobre SARS-CoV-1 e MERS-CoV sugeriram que gestantes estariam em maior risco da severidade da doença, morbidade ou mortalidade em comparação com a população em geral. SARS-CoV-2 está relacionado geneticamente ao SARS-CoV-1 e utiliza a Enzima Conversora de Angiotensina 2 humana (ECA2) como receptor das células hospedeiras. A interação entre SARS-CoV-2 e ECA2 aumenta a produção de citocinas inflamatórias envolvidas com lesão pulmonar grave e resultados clínicos preocupantes em pacientes com COVID-19 (KARIMI-ZARCHIA M, et al., 2020).

COVID-19 tem semelhança à gripe comum com sintomas como febre, tosse, infecção do trato respiratório inferior até casos de pneumonia grave com Síndrome do Desconforto Respiratório Agudo (SDRA) (GUAN WJ, et al., 2020). Anosmia (perda de olfato) e disgeusia (perda de paladar) são sintomas precoces e há também casos de portadores assintomáticos. A maioria das mortes ocorre devido à SDRA e à falência múltipla dos órgãos. Alta morbimortalidade tem sido observada em idosos e pacientes com comorbidades (ZHOU F, et al., 2020).

A transmissão do SARS-CoV-2 ocorre principalmente de pessoa para pessoa através de gotículas respiratórias e pode ser detectado em outros fluidos biológicos, como lavado broncoalveolar, sangue e saliva. Ainda existem lacunas consideráveis no que diz respeito à presença de SARS-CoV-2 nas fezes, na urina e no leite materno (CHAN JFW, et al., 2020).

Estudos epidemiológicos afirmam que pessoas de qualquer idade correm o risco de infecção pelo SARSCoV-2 e a gravidade está associada à idade e às comorbidades. As gestantes devem ser levadas em consideração nas políticas e estratégias de saúde pública com foco na prevenção e no tratamento da infecção viral. Como os dados epidemiológicos da COVID-19 vêm evoluindo rapidamente, os neonatologistas precisam atualizar continuamente as estratégias de manejo de recém-nascidos de mães suspeitas ou positivas para esta doença (WU Z e MCGOOGAN JM, 2020).

Grávidas são geralmente os membros mais vulneráveis da sociedade durante um surto de uma doença infecciosa. Alterações fisiológicas e imunológicas que acompanham a gravidez podem aumentar a suscetibilidade ao patógeno viral recém-emergente e a gravidade da infecção. Doenças virais novas e reemergentes, como hepatite $\mathrm{E}$, influenza e varicela, demonstraram ter um curso clínico mais grave, maiores taxas de complicações e de letalidade entre mulheres grávidas do que em não-grávidas (SAPPENFIELD E, et al., 2013).

Durante a pandemia de influenza H1N1 de 2009, grávidas apresentaram um maior risco de pneumonia grave, SDRA, ventilação mecânica e óbito quando comparadas com não grávidas em idade reprodutiva. Embora a maioria das infecções por coronavírus humano seja leve, as epidemias severas de SARS e 
MERS obtiveram resultados semelhantes e pacientes grávidas demonstraram ser mais propensas a desenvolver disfunção de órgãos e chegar ao óbito. Cogita-se, então, que gestantes podem ser mais suscetíveis à infecção por COVID-19 do que a população em geral (LUO Y e YIN K, 2020).

Nos países que optaram pelo plano de contenção nacional, as atividades médica e cirúrgica não emergenciais estão suspensas e os serviços obstétricos experimentam um aumento nas gestações "em risco".

As grávidas não são reconhecidas como população em alto risco; no entanto, elas ainda podem estar vulneráveis aos riscos médicos e sociais. Além dos riscos específicos do vírus, doenças com febre alta no início da gravidez estão associadas a defeitos congênitos específicos, como defeitos do tubo neural (BUEKENS P, et al., 2020).

As manifestações clínicas das pacientes grávidas com COVID-19 variam de assintomáticas a sintomas muito graves, semelhantes ao que ocorre em pacientes não grávidas. Febre e fadiga têm sido os principais sintomas e os menos comuns são dor de garganta e falta de ar. Atualmente, não se sabe se o vírus da COVID-19 pode ser transmitido através do leite materno, porém sabe-se que uma mãe infectada pode transmitir o vírus através de gotículas respiratórias durante a amamentação (RASMUSSEN SA, et al., 2020).

Estudos que avaliam efeitos da desregulação da resposta inflamatória em mulheres grávidas com SARSCoV-2, consequências sobre fisiopatologia e função genômica da placenta e potenciais efeitos a curto e longo prazo nos resultados do desenvolvimento infantil ainda não foram totalmente averiguados.

Gestantes infectadas podem apresentar níveis mais elevados de interleucina IL-6 em comparação com não grávidas, o que pode ter implicações importantes no desenvolvimento fetal. Portanto, supõe-se que tempestade de citocinas e hiperinflamação em gestantes com SARS-CoV-2 podem aumentar riscos de distúrbios do desenvolvimento neurológico em neonatos (YIN M, et al., 2020).

Mulheres com algum ou nenhum sintoma da COVID-19 não necessitam de tratamento de rotina ou atendimento hospitalar, apenas precisam ser monitoradas por até 15 dias para evidências de deterioração respiratória. Amostras de sangue coletadas durante o pré-natal ou durante o parto podem ser utilizadas para gerar estimativas populacionais de soroprevalência de doenças infecciosas. Além do atendimento prénatal, as amostras podem ser coletadas durante o período da pandemia de SARS-CoV-2.

Amostras coletadas antes e após a circulação viral podem trazer uma estimativa de infecções anteriores e uma melhor compreensão da epidemiologia das gestações afetadas. Na ausência de tratamento específico eficaz, a abordagem primária à terapia para as gestantes é principalmente sintomática e, em casos de desconforto respiratório crítico, é considerado maximizar a capacidade pulmonar de oxigenação e podem receber tratamento antiviral para melhorar sua condição clínica (YIN M, et al., 2020).

Esta revisão narrativa de literatura traz informações sobre relação entre gestação e COVID-19 destacando a consequência dos níveis aumentados da ECA2 durante a gravidez, papel do sistema imunológico materno frente a esta patologia, manejo e medidas de controle de infecção de COVID-19 na gravidez e riscos da amamentação em gestantes com COVID-19. Pesquisas foram feitas nas bases de dados PubMed e Science Direct voltadas para publicação de artigos em inglês que retrataram sobre infecções virais, COVID-19, gestação e amamentação.

\section{REVISÃO BIBLIOGRÁFICA}

\section{COVID-19 e Gravidez}

Na epidemia de SARS em 2002/2003, os resultados clínicos foram piores em grávidas do que em não grávidas, inclusive taxas crescentes de nascimentos prematuros e de abortos foram associadas a infecções por SARS-CoV-1. Cerca de $50 \%$ das gestantes infectadas com SARS demandaram cuidados intensivos e $33 \%$ delas necessitaram de ventilação mecânica. A taxa de mortalidade de grávidas por SARS atingiu 25\% (WONG SF, et al., 2004). As características clínicas em grávidas com COVID-19 são semelhantes às não grávidas infectadas. 
Uma investigação conjunta realizada pela OMS e China avaliou 147 mulheres grávidas chinesas (64 casos confirmados, 82 suspeitos de COVID-19 e um assintomático), das quais $8 \%$ delas apresentaram sintomas graves. Concluiu-se, então, que grávidas com COVID-19 não apresentavam maior risco de desenvolver sintomas graves (ZHU H, et al., 2020).

Não há relatos de que SARS-CoV-2 tenha sido detectado nos líquidos amniótico, placentário e no sangue do cordão umbilical, porém a transmissão vertical em potencial não pode ser descartada. Dong $L$, et al. (2020) descreveram um caso de recém-nascido, de mãe com COVID-19, com sorologia IgM e IgG positiva contra SARS-CoV-2 ao nascimento.

Embora a IgG possa ser secundária à transferência transplacentária, o recém-nascido também teve resultado positivo para anticorpo IgM contra SARS-CoV-2. Uma vez que IgM não atravessa a placenta, este resultado foi atribuído à uma exposição intra-uterina ao SARS-CoV-2. Todos os testes RT-PCR foram negativos. Portanto, tornam-se necessários dados abrangentes sobre a população de gestantes com COVID-19 para uma melhor compreensão acerca da transmissão vertical.

Levantaram-se algumas preocupações em relação ao potencial de infecção durante e após a gravidez, incluindo (a) presença de familiares devido às restrições de quarentena, (b) possível exposição ao SARSCoV-2 durante visitas médicas, (c) exigência de interrupção precoce da gravidez por meio de cesariana, (d) uso constante de hipoclorito de sódio e álcool etílico 70\% que podem exercer efeitos tóxicos, e (e) durante a fase de amamentação ou cuidados neonatais (RASHIDI FAKARI F e SIMBAR M, 2020).

Adicionalmente, a administração de medicamentos durante a gestação necessita de atenção médica contínua, levando-se em consideração a Farmacologia Fetal, pois é necessário reconhecer peculiaridades farmacocinéticas e farmacodinâmicas do feto, os processos de transporte placentário e analisar a relação da exposição aos fármacos (PEIXOTO S, 2014). Principalmente, tendo em vista que muitas substâncias presentes em medicamentos são teratogênicas, o que implica em alterações genéticas, deficiências física e/ou mental ao longo da vida (DIAV-CITRIN O e KOREN G, 2000).

Até o momento, não há estudos sobre casos graves de COVID-19 e complicações obstétricas durante o primeiro trimestre de gestação. Ademais, os dados da atual pandemia devem ser considerados para 0 gerenciamento de infecções por COVID-19 durante a gravidez, pois o curso clínico dessa doença e a resposta aos tratamentos parecem diferir daqueles dos surtos anteriores envolvendo outros coronavírus (LIANG H e ACHARYA G, 2020). Mais estudos são necessários para entender a patogênese e a epidemiologia da SARS-CoV-2 durante a gravidez, incluindo aspectos como o tempo de infecção materna, idade gestacional, efeitos das comorbidades e frequências de resultados adversos.

\section{Expressão aumentada de ECA2 durante a gravidez}

A ECA é parte central do sistema renina-angiotensina (SRA) sendo um importante regulador da pressão arterial em mamíferos. A ECA2, por sua vez, é uma peptidase que cliva resíduos específicos da Angiotensina I (Ang I) para formar os peptídeos ativos com diversos efeitos fisiológicos Angiotensina II (Ang II), Ang 1-9 e Ang 1-7. Este último apresenta atividades vasodilatadora, anti-trombótica e anti-inflamatória e neutraliza os efeitos contráteis de Ang II; portanto, acredita-se que a ECA2 module o efeito do SRA durante a tensão vascular (DONOGHUE M, et al., 2000).

O perfil hormonal de uma gestação normal é caracterizado por um aumento precoce de todos os componentes do Sistema Renina Angiotensina Aldosterona (SRAA), incluindo ECA2. Isso aumenta a possibilidade de as gestantes estarem em maior risco de infecção por SARS-CoV-2. Além disso, a baixa pressão arterial em grávidas é mantida através de um equilíbrio entre ser resistente aos efeitos pressores da Ang II e aumentar níveis de Ang 1-7, exibindo respostas vasodilatadoras sistêmicas.

Na pré-eclâmpsia, esse equilíbrio é perdido com uma resposta exagerada à pressão arterial de Ang II. A pré-eclâmpsia também está associada à diminuição de Ang 1-7 no plasma materno. Como o SARS-CoV-2 se liga à ECA2 e causa regulação negativa no SRA, as infecções virais acometidas durante a gravidez podem potencializar anormalidades no SRAA, ou seja, aumentar Ang II e diminuir Ang 1-7 (GAROVIC VD, et al., 2020). 
COVID 19 e pré-eclâmpsia compartilham mecanismos incluindo disfunção de células endoteliais e anormalidades da coagulação. Como a característica da pré-eclâmpsia é a disfunção endotelial, a infecção por SARS-CoV-2 durante a gravidez pode reproduzir a disfunção microvascular, causando endotelite. Inflamação sistêmica e disfunção microcirculatória, caracterizadas pela vasoconstrição e isquemia, podem contribuir para um estado de pró-coagulopatia, como demonstrado por altas taxas de trombose venosa profunda, acidente vascular cerebral e embolia pulmonar, os quais são relatados em pacientes com COVID19 (XIE Y, et al., 2020).

Ativação do sistema complemento na pré-eclâmpsia e na COVID-19 pode resultar em lesão vascular trombótica grave quando esses dois estados de doença estão presentes simultaneamente. Portanto, anormalidades do SRAA, disfunção endotelial, ativação do complemento e os efeitos pró-coagulopáticos da COVID-19 são semelhantes aos que ocorrem em gestações pré-eclampticas, resultando potencialmente em dano vascular progressivo (BAMPOE S, et al., 2020).

Gravidez e suas complicações representam um estado vulnerável à infecção invasiva por SARS-CoV-2, refletindo vários mecanismos celulares sobrepostos. Além do efeito citotóxico direto do vírus, a lesão tecidual na COVID-19 é mediada por uma resposta inflamatória excessiva, comumente referida como tempestade de citocinas. A tempestade de citocinas é mediada por respostas imunes significativamente modificadas durante a gestação e pode contribuir para as características clínicas e laboratoriais da COVID19 durante a gravidez (NARANG K, et al., 2020).

\section{$O$ papel da resposta imune materna à COVID-19}

O sistema imunológico materno estabelece e mantém a tolerância ao feto alogênico, preservando a capacidade de proteção contra agentes microbianos. Uma gravidez bem-sucedida depende de adaptações imunes sistêmica e localmente reguladas. Em vez de manter a supressão imunológica, o estado imunológico materno muda ativamente com o crescimento e o desenvolvimento fetal em diferentes estágios gestacionais: de um estado pró-inflamatório (benéfico para a implantação e placentação do embrião) no primeiro trimestre a um estado anti-inflamatório (útil para o crescimento fetal) no segundo trimestre e, finalmente, atingindo um segundo estado pró-inflamatório (preparando-se para o início do parto) no terceiro trimestre (AGHAEEPOUR N, et al., 2017).

O sistema imunológico de gestantes é preparado para defender a invasão de patógenos. Células imunes inatas, como as Natural Killers e os monócitos, respondem mais fortemente às infecções virais, enquanto algumas respostas adaptativas são reguladas negativamente durante a gravidez, como por exemplo no número diminuído de células $\mathrm{T}$ e $\mathrm{B}$. Adicionalmente, durante a gravidez, o trato respiratório superior tende a ficar inchado devido a um alto nível de estrogênio e progesterona, e a expansão pulmonar restrita torna a gestante suscetível a patógenos respiratórios.

Estudos mostraram que a infecção por SARS durante a gravidez pode aumentar taxas de aborto espontâneo, parto prematuro e restrição de crescimento intra-uterino e essas complicações podem ter sido causadas pelo efeito direto do vírus (WONG SF, et al., 2004).

Em casos graves, a infecção por COVID-19 está associada a uma tempestade de citocinas caracterizada por concentrações plasmáticas aumentadas de interleucinas 2 (IL-2), IL-7, IL-10, do fator estimulador de colônias de granulócitos, da proteína inflamatória de macrófagos-1a e do fator de necrose tumoral alfa. Com base no conhecimento de que grávidas no primeiro e terceiro trimestres estão no estado pró-inflamatório, a tempestade de citocinas induzida pelo SARS-CoV-2 pode provocar um estado inflamatório mais grave nessas mulheres. Ademais, a ocorrência de inflamação como resultado de infecção viral pode afetar vários aspectos do desenvolvimento cerebral fetal e levar a uma ampla gama de disfunções neuronais e fenótipos comportamentais que são reconhecidos posteriormente na vida pós-natal (LIU H, et al., 2020).

O impacto da COVID-19 em gestantes atraiu muita atenção em relação à morbidade, mortalidade e resultados perinatais. Os sintomas iniciais dos recém-nascidos infectados pelo SARS-CoV-2 são falta de ar, aumento da frequência cardíaca, vômitos e erupções cutâneas pelo corpo (FAVRE G, et al., 2020), seguido posteriormente por trombocitopenia com função hepática anormal, hemorragia gastrointestinal, choque refratário e coagulação intravascular disseminada, podendo chegar a óbito (ZHU H, et al., 2020). 


\section{Manejo e medidas de controle de infecção de COVID-19 na gravidez}

Os princípios para manejo incluem isolamento social, procedimentos de controle de infecções, testes para diagnóstico de infecção e coinfecção por SARS-CoV-2, oxigenoterapia, prevenção da sobrecarga de fluidos, terapia empírica com antibióticos (devido ao risco secundário de infecção bacteriana), monitoramento das contrações fetal e uterina, ventilação mecânica precoce para insuficiência respiratória progressiva, planejamento de parto individualizado e abordagem de equipes com consultas multi especializadas.

Fornecer uma vigilância para detecção precoce de uma piora do curso materno da doença, bem como monitorar evidências de complicações obstétricas (trabalho de parto prematuro ou comprometimento fetal) também são necessárias (LIU H, et al., 2020).

Medidas simples podem aprimorar a ventilação em gestantes com COVID-19, mesmo naquelas graves com SDRA. A administração conjunta de oxigênio nasal de alto fluxo com a posição prona e lateralização otimizam a função respiratória e prolongam a gravidez, o que evita prematuridade extrema. Logo, essa combinação é uma estratégia prática e eficaz para evitar a intubação em gestantes. Contudo, em caso de instabilidade hemodinâmica a ventilação mecânica é a medida mais prudente tanto para mãe quanto para o feto, ressaltando que o parto em si não traz benefícios diretos para a mãe, enquanto, a continuação da gestação é essencial para o desenvolvimento do embrião (VIBERT F, et al., 2020).

Alterações na frequência cardíaca fetal podem ser uma preliminar de desgaste respiratório materno. Fundamentado na experiência com SARS e MERS, a ventilação mecânica pode não ser suficiente para suprir a oxigenação adequada. Caso isto ocorra, é aconselhável oxigenação por membrana extracorpórea que deve ocorrer apenas em centros médicos experientes. Decisões sobre o parto devem considerar a idade gestacional do feto e devem ser tomadas em conjunto com o pediatra neonatologista (PACHECO LD, et al., 2018).

A detecção e intervenção precoces da COVID-19 podem reduzir possíveis complicações obstétricas, como aborto espontâneo, restrição de crescimento intra-uterino e parto prematuro. Além disso, a intervenção medicamentosa nestas pacientes pode ser alterada pelas inúmeras mudanças fisiológicas como dos níveis hormonais, volume de líquidos e gorduras, taxa de filtração glomerular e de enzimas metabolizadoras de medicamentos, o que afeta substancialmente a farmacocinética e a eficácia do medicamento e sua toxicidade (PINHEIRO EA e STIKA CS, 2020).

Não há tratamento antiviral específico às grávidas. Embora estudos pré-clínicos e de transferência placentária sejam necessários para os potenciais medicamentos anti-SARS-CoV-2, alguns fármacos podem ser utilizados nas gestantes. Interferon, hidroxicloroquina e lopinavir/ritonavir têm resultados promissores, pois são utilizados em outras patologias. Há confirmações sobre o uso seguro, durante a gestação, da colchicina, de esteróides (como a metilprednisona e dexametasona), de oseltamivir, de azitromicina e de alguns monoclonais.

Entretanto, alguns medicamentos são proibidos na gravidez devido à teratogenicidade conhecida (talidomida) ou toxicidade fetal (bloqueadores do SRA). Ainda não há estudos sobre o uso seguro do remdesivir em mulheres grávidas, e, portanto, continua sob investigação devido à sua toxicidade (hipotensão e disfunções renais e hepática). Outros medicamentos, incluindo inibidores da IL-6 (umifenovir e favipiravir) e alguns antiparasitários têm informações inconsistentes sobre suas aplicações às gestantes (LOUCHET M, et al., 2020).

O tratamento é iniciado quando possíveis benefícios superam riscos da terapia ao feto. Mesmo após o controle da infecção, o desenvolvimento intrauterino fetal deve ser monitorado, pois a inflamação da placenta pode persistir por um período prolongado. Grávidas com COVID-19 devem ser monitoradas durante a gestação e no pós-parto, pois receberam terapia medicamentosa antiviral e exposição à radiação de exames tomográficos. Apesar do tabu envolvendo exames radiológicos em gestantes, a quantidade de radiação nesses procedimentos não apresenta riscos de malformações, aborto espontâneo ou morte fetal (GUILBAUD L, et al., 2019). 
Para gestantes com a COVID-19, recomenda-se que o parto deve ser determinado pela indicação obstétrica e não deve ser influenciado pela presença do SARS-CoV-2, a menos que a condição respiratória da gestante exija intervenção urgente para evitar complicações no momento do parto (https://www.rcog.org.uk/en/guidelines-research-services/guidelines/coronavirus-pregnancy/---06-july-2020).

A transmissão vertical pode ocorrer durante o parto vaginal através do contato do neonatal com o reto. Diante de tal situação, um estudo propôs medidas preventivas adicionais àquelas propostas pelas associações internacionais a serem tomadas como um esforço de reduzir o potencial risco de transmissão vertical do SARS-CoV-2 durante o parto vaginal.

Dentre essas medidas preventivas destaca-se o exame de swab realizado no reto e nas fezes e a higiene adequada da área genito-anal que deve ser feita, usando compressas quentes embebidas em desinfetante e aplicando um curativo anoperineal discreto para cobrir o ânus antes da fase de expulsão do bebê (CAROSSO A, et al., 2020).

\section{Amamentação em gestantes com COVID-19}

Leite materno fortalece o sistema imunológico dos bebês na prevenção de infecções e as mães que amamentam têm riscos reduzidos de hipertensão e de câncer de mama (SCHWARZ EB e NOTHNAGLE M, 2015). Há falta de consenso entre as autoridades de saúde em relação à amamentação para mulheres com COVID-19. Em fevereiro de 2020, a Comissão Nacional de Saúde da China recomendou que neonatos de mães com suspeita ou confirmação de COVID-19 fossem isolados por pelo menos 14 dias e que não fossem amamentados devido ao risco de infecção (WANG W, et al., 2020).

A OMS aconselha que a amamentação seja determinada pela mãe em concordância com a família e os profissionais de saúde e todas as medidas de prevenção para evitar a propagação do vírus ao bebê devem ser tomadas, inclusive usar máscaras e lavar regularmente as mãos e os seios antes da amamentação (WHO, 2020). Caso a mulher opte por extrair o leite materno, recomendações para limpar bombas e utensílios durante e após cada uso devem ser seguidas.

É possível que uma pessoa saudável e treinamento qualificado forneça leite materno ao bebê usando copo ou colher. Se não houver produção de leite materno, um banco de leite pode ser contatado. A possibilidade de excreção de drogas para o leite materno, com possíveis efeitos adversos aos recémnascidos, ainda precisa ser investigada. Até o momento, não há evidências sobre a presença de SARSCoV-2 no leite materno de grávidas com COVID-19 (ANDERSON PO, 2020).

\section{CONSIDERAÇÕES FINAIS}

Estratégias de vigilância sanitária, pesquisas sobre soroprevalência e pesquisas socioeconômicas precisam ser implementadas para abordar as lacunas de conhecimento que envolvem o efeito da COVID-19 na gravidez. A alavancagem dos registros de gravidez existentes e a vigilância longitudinal vinculada à mãe e o bebê para capturar informações clínicas e de resultados sobre a COVID-19 podem ajudar a facilitar a rápida coleta de dados para as ações de saúde pública. Estudos de base populacional podem elucidar riscos de infecção e se isto varia de acordo com a idade gestacional ou outros fatores médicos e sociais. Assim que novos tratamentos e vacina estiverem disponíveis, uma rede global de pesquisa será crucial na avaliação do seu uso entre gestantes.

\section{REFERÊNCIAS}

1. AGHAEEPOUR N, et al. An immune clock of human pregnancy. Sci Immunol, 2017; 2(15): eaan2946.

2. ANDERSON PO. Breastfeeding and respiratory antivirals: coronavirus and influenza. Breastfeed Med, 2020; 15(3): 128.

3. BAMPOE S, et al. Novel coronavirus SARS-CoV-2 and COVID-19. Practice recommendations for obstetric anaesthesia: what we have learned thus far. Int J Obstet Anesth, 2020; 43: 1-8.

4. BUEKENS $P$, et al. A call for action for COVID-19 surveillance and research during pregnancy. Lancet Glob Health, 2020; 8(7): e877-e878. 
5. CAROSSO A, et al. How to reduce the potential risk of vertical transmission of SARS-CoV-2 during vaginal delivery? Eur J Obstet Gynecol, 2020; 250: 246-249.

6. CHAN JFW, et al. A familial cluster of pneumonia associated with the 2019 novel coronavirus indicating person-toperson transmission: a study of a family cluster. Lancet, 2020; 395(10223): 514-23.

7. DIAV-CITRIN O, KOREN G. Human teratogens: a critical evaluation. Motherisk: 2000. ISBN: 0-9686598-1-0.

8. DONG L, et al. Possible vertical transmission of SARS-CoV-2 from an infected mother to her newborn. JAMA, 2020; 323(18): 1846-1848.

9. DONOGHUE $M$, et al. A novel angiotensin-converting enzyme-related carboxypeptidase (ACE2) converts angiotensin I to angiotensin 1-9. Circ Res, 2000; 87(5): e1-e9.

10. FAVRE G, et al. 2019-nCoV epidemic: what about pregnancies? Lancet, 2020; 395(10224): e40.

11. GAROVIC VD, et al. Incidence and long-term outcomes of hypertensive disorders of pregnancy. J Am Coll Cardiol, 2020; 75: 2323-2334.

12. GUAN WJ, et al. Clinical characteristics of coronavirus disease 2019 in China. N Engl J Med, 2020; 382: $1708-1720$.

13. GUILBAUD L, et al. Pregnancy outcome after first trimester exposure to ionizing radiations. Eur J Obstet Gynecol Reprod Biol, 2019; 232: 18-21.

14. KARIMI-ZARCHIA M, et al. Vertical Transmission of Coronavirus Disease 19 (COVID-19) from Infected Pregnant Mothers to Neonates: A Review. Fetal Pediatr Pathol, 2020; 39(3): 246-250.

15. LIANG H, ACHARYA G. Novel coronavirus disease (COVID-19) in pregnancy: What clinical recommendations to follow? Acta Obstet Gynecol Scand, 2020; 99: 439-442.

16. LIU H, et al. Why are pregnant women susceptible to COVID-19? An immunological viewpoint. J Reprod Immunol, 2020; 139: 103122.

17. LOUCHET M, et al. Placental transfer and safety in pregnancy of medications under investigation to treat COVID-19. Am J Obstet Gynecol, 2020. [ahead of print]

18. LUO Y, YIN K. Management of pregnant women infected with COVID-19. Lancet Infect Dis, 2020; 20(5): 513-514.

19. NARANG K, et al. SARS-CoV-2 infection and COVID-19 during pregnancy: a multidisciplinary review. Mayo Clin Proc, 2020. https://doi.org/10.1016/j.mayocp.2020.05.011. [Epub ahead of print].

20. PACHECO LD, et al. Extracorporeal membrane oxygenation (ECMO) during pregnancy and postpartum. Semin Perinatol, 2018; 42: 21-25.

21. PEIXOTO, S. Manual de assistência pré-natal. Federação Brasileira das Associações de Ginecologia e Obstetrícia (FEBRASGO). 2nd ed. São Paulo. 2014.

22. PINHEIRO EA, STIKA CS. Drugs in Pregnancy: Pharmacologic and Physiologic Changes that Affect Clinical Care. Semin Perinatol, 2020; 44(3): 151221.

23. RASMUSSEN SA, et al. Coronavirus Disease 2019 (COVID-19) and pregnancy: what obstetricians need to know. Am J Obstet Gynecol, 2020; 222(5): 415-426.

24. RASHIDI FAKARI F, SIMBAR M. Coronavirus pandemic and worries during pregnancy. Arch Acad Emerg Med, 2020; 8: e21.

25. SAPPENFIELD E, et al. Pregnancy and susceptibility to infectious diseases. Infect Dis Obstet Gynecol, 2013; 2013: 752852.

26. SCHWARZ EB, NOTHNAGLE M. The maternal health benefits of breastfeeding. Am Fam Physician, 2015; 91(9): 603-604.

27. VIBERT F, et al. Prone positioning and high-flow oxygen improved respiratory function in a 25 -week pregnant woman with COVID-19. Eur J Obstet Gynecol Reprod Biol, 2020; 250: 257-258.

28. WANG W, et al. Detection of SARS-CoV-2 in different types of clinical specimens. JAMA, 2020; 323(18): 1843-1844.

29. WONG SF, et al. Pregnancy and perinatal outcomes of women with severe acute respiratory syndrome. Am J Obstet Gynecol, 2004; 191: 292-297.

30. WORLD HEALTH ORGANIZATION (WHO). Director-General's opening remarks at the media briefing on COVID-19. Geneva: WHO; 2020. [cited 2020 Mar 11]. Available from: https://www.who.int/dg/speeches/detail/who-directorgeneral-s-openingremarks-at-the-media-briefing-on-covid-19---11-march-2020.

31. WORLD HEALTH ORGANIZATION (WHO). Home care for patients with COVID-19 presenting with mild symptoms and management of their contacts. Interim guidance. Geneva: WHO; 2020 [cited 2020 Mar 17]. Available from: https://www.who.int/publications-detail/home-care-for-patients-with-suspected-novel-coronavirus-(ncov)-infection.

32. WU Z, MCGOOGAN JM. Characteristics of and important lessons from the coronavirus disease 2019 (COVID-19) outbreak in China: summary of a report of 72314 cases from the Chinese Center for Disease Control and Prevention. JAMA, 2020; 323(13): 1239-1242.

33. XIE Y, et al. COVID-19 complicated by acute pulmonary embolism. Radiol Cardiothorac Imaging, $2020 ; 2$ : e200067.

34. YIN M, et al. Severe acute respiratory syndrome coronavirus 2 (SARS-CoV-2) infection during pregnancy in China: a retrospective cohort study. MedRxiv, 2020.

35. ZHOU F, et al. Clinical course and risk factors for mortality of adult inpatients with COVID-19 in Wuhan, China: A retrospective cohort study. Lancet, 2020; 395: 1054-1062.

36. ZHU H, et al. Clinical analysis of 10 neonates born to mothers with 2019-nCoV pneumonia. Transl Pediatr, 2020; 9(1): 51-60. 